\title{
The Study of How Open Community Based on Combination Weighting Method Influence on Road Capacity
}

\author{
Zhuhui $\mathrm{He}^{1}$, Rui Liao ${ }^{2}$ \& Longlong Wang ${ }^{1}$ \\ ${ }^{1}$ Electrical and Information College, Jinan University, China \\ ${ }^{2}$ Finance Department of International Business School, Jinan University, China \\ Correspondence: Zhuhui He, Jinan University, No. 206, Qianshan Road, Xiangzhou District, Zhuhai, China. Tel: \\ 0086-137-1348-1021.E-mail: 516183810@qq.com
}

Received: March 5, 2017

doi:10.5539/mas.v11n6p15
Accepted: March 30, 2017

Online Published: April 17, 2017

\begin{abstract}
The paper studies whether the opening of community can improve the road capacity. First of all, based on the consideration of road space and traffic quality, the evaluation indexes are: road network density, road network connectivity, non-linear coefficient, traffic volume of main road beside community, delay time of intersection, travel time consumption and vehicle accessibility. Secondly, the weight of the index is calculated by the method of relative comparison, the variation coefficient method and the entropy weight method. Then, we sort the three weights, using the correlated coefficient and degree of spearman rank to combine the three weights, so as to get combined weights of various index and achieve the effect which makes good use of the method. Finally, we combine indicators and weights, establish a comprehensive evaluation model, and assess the road capacity before and after the opening of the road. Through the calculation, it is found that the comprehensive evaluation index after the opening of the community is higher than that before the opening, that is, the positive impact on the surrounding roads.
\end{abstract}

Keywords: combination weighting method, open community, road space, traffic quality

\section{Introduction}

Most of the living community of modern cities are mainly closed-type areas. The so-called closed-type community refers to the use of fully enclosed management mode, so that the use of planning elements such as greening, roads and public facilities are independent from the urban structure, which is a self-contained system, to meet the needs of the communities. This article focuses on the closeness of the road system to the outside world, but with the economic developing and the spirit of the residents improving, the shortcomings of closed-end community gradually emerge. As the road system of the closed-type community is closed-planning, the city's original structure of road network has been destroyed, which makes people queue for more time, likely to cause traffic jam, and is not benefit to urban traffic development, as well as increase the difficulty of residents travel. China's State Council issued a regulation on the promotion of street regulations, abolishing closed residential community, open residential communities and unit courtyards, which triggered widespread concern and discussion. The focus to the discussion is on the possible passage problem of roads in open areas, such as whether the open community can optimize the road network structure, improve road capacity and improve traffic conditions.

It is important to study whether the road capacity can be improved before and after the opening of the closed community for urban road planning. Zhan Bin, Cai Ruidong and Hu Yuancheng(2016), research respectively from the openness degree of open community, community location, and size, establish connection model between open community and urban road network for different communities to develop appropriate open strategy. Ma Xiaofeng (2013), Shang Yuhang (2015) analyze the openness problem from the aspect of openness possibility and community design after openness. Yan Zi-an, Dong Jun-yan (2017) using the analytic hierarchy process to evaluate the road traffic capacity before and after the opening of the district, it is concluded that the opening of the district can alleviate the traffic pressure of the surrounding vehicles. Yu Xiaoyu(2017)introduces the dimensionless road network parameters and road network resistance, then gets the relationship between the road network resistance and the inherent attributes and traffic flow of the road network, as well as how different types of community opening influence on road traffic. Zhang Penghao, Xu Yanhai(2016) using NS cellular 
automata to simulate the vehicles through the district, make a conclusion from the microscopic point of view that open community can alleviate the surrounding road pressure.

This paper refers to the "Specifications of Urban Road Traffic Planning and Design ", respectively, taking the road space and traffic quality into consider, and selects the road space indicators reflect the road network density, road network connectivity and non-linear coefficient. Also the traffic quality indicators such as trunk traffic flow, delay time of intersection, travel time consumption and accessibility. And then use the combination weighting method based on spearman rank correlation coefficient and correlation degree to realize three methods be complemented each other, that is, relative comparison method, entropy weight method and coefficient of variation method. According to the combined weight and the respective index, the comprehensive evaluation model is established, and we can obtain the change of comprehensive score of the surrounding road traffic capacity before and after the opening of the community.

\section{Evaluation Model of Road Capacity Based on Combination Weighting Method}

\subsection{The Establishment of Index Evaluation System}

\subsubsection{The Selection of Index}

In order to establish a reasonable comprehensive evaluation index system, it is necessary to select the indicators which can reflect the impact of the open area on the surrounding roads according to the functions of the traffic branch network and the "Specification of Urban Road Traffic Planning and Design". In order to be able to synthesize the advantages of various weights method, we choose the combination weighting method based on the correlation coefficient, establish a comprehensive evaluation index system, and deal with the index value by the appropriate data normalization algorithm, and then get the road comprehensive score. According to the road network specifications, select the spatial indicators 3: road network density, road network connectivity, non-linear coefficient, besides, traffic quality indicators 4 : the main road traffic around the community, delay time of intersection, travel time assumptions and accessibility.

\subsubsection{Quantification of Indicators}

(1) Road Network Density

Openness increase the number of urban roads. In order to quantitatively measure the changes in this indicator, we introduce road network density $\delta\left(\mathrm{km} / \mathrm{km}^{2}\right)$, which is, road length and built area ratio in the completion of the district, which road width is 3.5 meters, not include sidewalks.

$$
\delta=\frac{\sum L_{i}}{A}
$$

Among them, $L_{i}$ represents road length $(\mathrm{km})$ in respective levels of city road network.

\section{(2) Road Network Connectivity}

Road network connectivity $J$ is the indicator related total number of nodes and the total number of road network related, which is from the macro reflect the maturity of the network. The formula:

$$
J=\frac{\sum_{i=1}^{n} m_{i}}{N}=\frac{2 M}{N}
$$

Among them, $\mathrm{N}$ is the number of road network nodes, $m_{i}$ is the side number of $l$ th node, $M$ is the total side number of road network. The higher the $J$ value, the less breakage roads, the better into the ring or into the network, otherwise the worse.

\section{(3)Non-Linear Coefficient}

The non-linear coefficient is the ratio of the actual road length between two nodes in the network and the straight line distance between two points. It is used to judge whether the layout of the road network is reasonable and the degree of linearity is one of the important indexes to evaluate whether the form of road network is reasonable. In general, the degree of linearity is measured by a non-linear coefficient $(K)$ :

$$
K=L_{i j} / L_{i j}^{\prime}
$$


Among them, $L_{i j}$ is the actual distance between two points, $L_{i j}^{\prime}$ is the straight line distance. In those cities unlimited in geographical conditions, non-linear coefficient should be controlled under 1.3.

(4) The Main Road Traffic Around the Community

Traffic flow, setting the vehicles through a specific section by unit time as a standard, shows the number of vehicles through the road in a certain period of time. Traffic flow in the main road around the district before and after the opening of the community is different, that is why it can indicate road capacity. Traffic flow = unit time * speed / (car distance + body length)

$$
Q=\frac{t * v}{a+b}
$$

Among them, $Q$ is the traffic flow in the main road, $t$ is the unit time, $v$ is average speed, $a$ is the average value of car distance, $b$ is car body length.

(5) Intersection Load

After the opening of the district, it will increase the intersection in the road, the service level and evaluation of the intersection depend on the calculation of the intersection load:

$$
t_{d}=\frac{0.5 T\left(1-\frac{t_{g}}{T}\right)}{1-\left[\min (1, \mathrm{x}) \frac{t_{g}}{T}\right]}
$$

Among them, $t_{d}$ is the intersection load, $T$ is the cycle length of traffic light, $t_{g}$ is the valid green signal time, the group of traffic lane $V / X$ or saturation, $\mathrm{X}$ is the ratio of the maximum service traffic to the basic capacity under the ideal conditions.

(6) Travel Time Assumption

Community residents have a certain tolerance limit for daily travel time, as to response to the number of residents and patience index at the same time, we definite the indicators of travel time consumption as $T$, calculated as follows:

$$
\mathrm{T}=0.1382 \mathrm{p}_{\mathrm{p}}+0.000582 \mathrm{~A}_{\mathrm{a}}+33.071
$$

Among them, $\mathrm{T}$ is the maximum travel time consumption ( $90 \%$ travel time consumption) (min) that the residents of the community can accept, $p_{p}$ represents the community population, $A_{a}$ is the community area $\left(\mathrm{km}^{2}\right)$.

\section{(7) Accessibility}

The accessibility of the road network refers to the travel distance, the travel time or the cost of any destination started from any place in the planning area. It is an important criterion for judging the performance of the traffic system. It can be calculated from the distance and time, to reflect convenience of residents traveling, and changes in the capacity of the road system before and after the change. This indicator is indicated by the travel distance.

$$
\overline{\mathrm{D}}=\frac{2}{n(\mathrm{n}-1)} \sum_{i=1}^{n} \sum_{j=i+1}^{n} d_{i j}
$$

Among them, $\overline{\mathrm{D}}$ is accessibility, $d_{i j}$ is minimum average travel distance of node $i-j$ in network, $n$ is the 
number of nodes in the district. The higher the $\overline{\mathrm{D}}$, the worse the accessibility, otherwise the better of that.

\section{(8) Standardization of Data}

In the multi- index comprehensive evaluation model, due to the measurement unit, the intrinsic attribute, the level of magnitude of each index are in difference, it can't be directly integrated and compared. Therefore, in order to unifying standard, we must mix all the evaluation indexes to eliminate the influence of the dimension, then transform them into the standard index value with no dimension, no level of magnitude difference and consistent direction, after that we synthesize these indexes . The index is divided into three types, which is, positive indicators, reverse indicators, balance indicators, which are to be standardized.

If a indicator is required for the higher the better, such as connectivity of road network $J$, then

$$
u_{i j}=\frac{f_{i j}-\min _{i} f_{i j}}{\max _{i} f_{i j}-\min _{i} f_{i j}}
$$

If a indicator is required for the lower the better, such as non-linear coefficient $K$, the intersection of delay time $d$, travel time assumption $\mathrm{T}$ and accessibility $\mathrm{D}$, then

$$
u_{i j}=\frac{\max _{i} f_{i j}-f_{i j}}{\max _{i} f_{i j}-\min _{i} f_{i j}}
$$

If a indicator is required for the best in stability, such as road network density $\delta$ and traffic flow in main roads $Q$, then

$$
u_{i j}=\frac{f_{i j}}{\overline{f_{i j}}}
$$

Among them, $u_{i j}$ is the standardized value, $f_{i j}$ is the original value.

\subsection{The Process of Calculating Combination Weights}

(1) Use AHP to determine the subjective weight of the indicator

The method of AHP is applicable to decision-making problems that are more complex in construction and criterion and difficult to quantify. After constructing the index system, it is necessary to compare every two factors at the same level, and invite experts in relevant fields to score independently and repeatedly on the importance of each index in the criterion layer, then the judgment matrix can be constructed.

Among them, $e_{i j}>0 ; e_{i j}=1 / e_{j i}(i \neq j) ; e_{i i}=1(i, j=1,2, \ldots, m), e_{i j}$ is the importance degree of indicator $i$ to indicator $j$.

The weight calculation of AHP is based on the judgment matrix to calculate the importance weights of the elements of one layer relative to its upper layer. It can be attributed to the problem that calculate the maximum eigenvalues and eigenvectors of the judgment matrix. The formula is as follows:

$$
\begin{gathered}
\bar{W}_{i}=\sqrt[m]{\prod_{j=1}^{m} e_{i j}} ; i, j=1,2,3, \ldots, m \\
w_{i}^{(a)}=\frac{\bar{W}_{i}}{\sum_{j=1}^{m} \bar{W}_{j}}
\end{gathered}
$$

Therefore, $w^{(a)}=\left[w_{1}^{(a)}, w_{2}^{(a)}, \ldots, w_{m}^{(a)}\right]^{T}$ is the feature vectors that we calculate, that is, weight vector.

Finally, a consistency test is required to calculate the value of the random consistency ratio $\mathrm{CR}=\mathrm{CI} / \mathrm{RI}$. When $\mathrm{CR}$ $<0.10$, it is considered that the judgment matrix A-B has satisfactory consistency. Otherwise, it is necessary to adjust the judgment matrix so as to make it satisfactory.

(2) Entropy Method

Entropy method is an objective method of weighting, according to the variation degree of the indicators, using 
the information entropy to calculate the entropy weight of each index, then we can obtain more objective index weights. The information entropy formula of the $j t h$ index is:

$$
E_{j}=-(\operatorname{lnm})^{-1} \sum_{i=1}^{m} p_{i j} \ln p_{i j}(\mathrm{j}=1,2, \ldots, \mathrm{n})
$$

Among them, $m$ is the number of objects that be evaluated, $n$ is the number of evaluation number, and

$$
p_{i j}=\frac{u_{i j}}{\sum_{i=1}^{m} u_{i j}}
$$

The smaller the entropy of an index, the greater the degree of variation of the index value, the more information provided, the greater the effect of the index in the comprehensive evaluation, the greater the weight. So we can calculate weight of the $j$ th index $w_{j}$ by the information entropy, the formula is:

$$
w_{j}=\frac{1-E_{j}}{n-\sum_{j=1}^{n} E_{j}}
$$

\section{(3) The Method of Variation Coefficient}

The basic idea of the variation coefficient method is that in the multi-index comprehensive evaluation, if an indicator is larger in the variation degree of observations on all the evaluated objects, it is more difficult to reach the average level, and it can distinguish clearly the ability of each evaluated object, so the indicator should give a greater weight; otherwise, it should be given a smaller weight.

As respective index in the evaluation index system has different dimensions, it is not appropriate to compare the degree of difference directly. In order to eliminate the effects caused by different dimensions of the evaluation indexes, we need to use the variation coefficient of each index to measure the difference in the value of each index. The steps to determine the weights of the indicators by using the coefficient of variation are as follows:

Step 1: According to the index data of each factor, calculate the average and standard deviation of each indicator.

Step 2: According to the average and standard deviation of the indicators, the formulas of variation coefficient in each index is as follows:

$$
V_{j}=\frac{\sigma_{j}}{\bar{u}_{j}}(j=1,2, \cdots, n)
$$

Among them: $V_{j}$ is the Coefficient of variation of the jth index; $\sigma_{j}$ is the standard deviation of the $j$ th index;

$\overline{u_{j}}$ is the average of the $j t h$ index.

Step 3: According to the variation coefficient of each indicator, the weights formula of the indicators are as follows:

$$
w_{j}=\frac{V_{j}}{\sum_{j=1}^{n} V_{j}}
$$

Therein, $w_{j}$ is the weight of the $j$ th index.

\section{(4) Determination of the Combination Weighting Method}

Firstly, using the size of correlation coefficient of the spearman rank in the rank correlation theory, we pick out the reference column in the calculation of gray correlation degree, then the method of determining the weight is sorted according to the size of the correlation degree and normalized by the correlation degree. We obtain the 
weights of various methods in the combination weighting method, use the weights to weight the index weights obtained in every weighting methods, and finally get the weight of the combination weighting method. The implementation steps are as follows:

Step 1: According to each evaluation index, the weights of the AHP method, the entropy weight method and the variation coefficient method can be calculated.

Step 2: Sort the weights obtained by the various weighting methods respectively, and get the rank values.

Step 3: What we mainly concern is whether the standard of each weighting method is consistent when weights the same index, for this purpose, we calculate the Spearman rank correlation coefficient. The formula of correlation coefficient of Spearman rank is as follows:

$$
\begin{gathered}
r_{s \cdot i k}=1-6 \sum_{j=1}^{n} d_{i k j}^{2} /\left(\mathrm{n}^{3}-n\right) \\
\mathrm{d}_{i k j}^{2}=\left(\mathrm{y}_{i j}-\mathrm{y}_{k j}\right)^{2}(j=1,2 \cdots \mathrm{n} ; i \quad \mathrm{k}=1,2, \cdots m)
\end{gathered}
$$

$r_{s \cdot i k}$ is the Spearman rank correlation coefficient of the ith method and the kth method; $y_{k j}$ is the sorting value of the $j$ th index in the kth weighting method, and $m$ is the number of methods that determine the weights; $n$ is the number of the indexes.

The size of $r_{\text {s.ik }}$ reflects the method $i$ and method $\mathrm{k}$ whether they are high or low level in consistency criteria when weighting, the smaller of $r_{s \cdot i k}$ the worse, the bigger of $r_{s \cdot i k}$ the better. From the Spearman rank correlation coefficient, it can be seen that the weighting criteria of these methods are sometimes close and sometimes different. This shows that there are many relationships in different degree among the conclusions of each method. In order to optimize and sort these methods, we find the maximum one $r_{\max }=\max \left\{r_{s \cdot i k}\right\}$ in Spearman rank correlation coefficients, and obtain its corresponding method.

Step 4: Take the largest weight in Spearman correlation coefficient as reference column, and calculate the gray correlation degree of the other methods in regard to it $\vec{R}=\left(\mathrm{R}_{l 1}, \mathrm{R}_{l 2}, \ldots, \mathrm{R}_{l m}\right)$ (among them, $R_{l l}=1$ ),then we sort the weighting methods by the size of correlation degree, finally we will normalize $\vec{R}$ to obtain the weight vector $\vec{W}=\left(\mathrm{W}_{1}, \mathrm{~W}_{2}, \ldots, \mathrm{W}_{m}\right)$ of the combination weighting method. Among them,

$$
\mathrm{W}_{i}=R_{l i} / \sum_{i=1}^{m} R_{l i}(\mathrm{i}=1,2, \ldots, \mathrm{m})
$$

Step 5: According to the weight vector $\vec{W}$ of combination weighting method and the weight matrix of various weighting methods, we can get the combination weights, the formula is:

$$
\begin{aligned}
& W A=\left(\mathrm{W}_{1}, \mathrm{~W}_{2}, \ldots, \mathrm{W}_{m}\right)\left(\begin{array}{cccc}
w_{11} & w_{12} & \cdots & w_{1 n} \\
w_{21} & w_{22} & \cdots & w_{2 n} \\
\cdots & \cdots & & \\
w_{m 1} & w_{m 2} & \cdots & w_{m n}
\end{array}\right) \\
& =\left(\sum_{i=1}^{m} W_{1} w_{i 1}, \sum_{i=1}^{m} W_{2} w_{i 2}, \cdots \sum_{i=1}^{m} W_{n} w_{i n},\right)
\end{aligned}
$$

$w_{m n}$ is the weight of the nth indicator of the $m$ th weighting method.

\subsection{The establishment of a comprehensive evaluation index system}

Based on the obtained weighting values and the standardized index data, the comprehensive score of the traffic capacity surrounded the community is obtained by the following formula (Han, 2009): 


$$
F_{i}=\sum_{j=1}^{m} W A_{j} u_{i j}, i=1,2, \ldots, n
$$

Among them, $F_{i}$ is the comprehensive score of the $i$ th item; $W A_{j}$ is the combination weight of the $j$ th index; $u_{i j}$ is the standardized value of the $j$ th index of the ith item.

\section{The Analysis of Result in the Model}

\subsection{Objection Selection}

According to the actual situation, the paper selects a community A in Changsha. Latitude and longitude of community Ais $\left(28.2^{\circ} \mathrm{N}, 112.9^{\circ} \mathrm{E}\right)$. China as the object of the model, and evaluates the traffic capacity before and after the opening of the community A, then obtains the influence degree of the open area on the road traffic capacity. The satellite map of community A is shown in Figure 1.

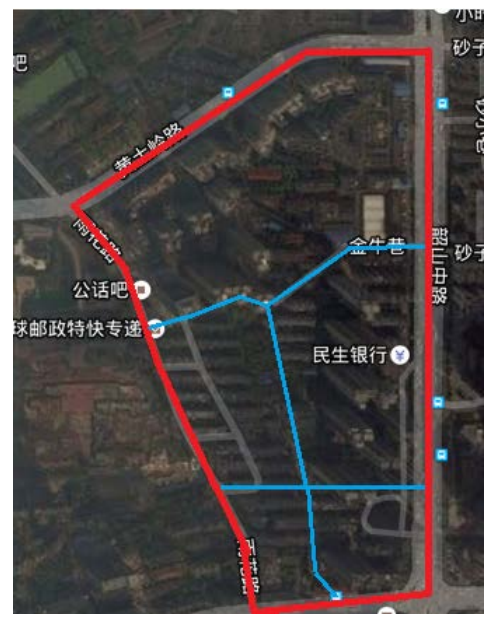

Figure 1. Changsha City, a Community A

\subsection{Quantitative Results of Indicators}

Through the measurement, statistics and calculation, we get the area of community A, the length of the road and other condition before closed and after the open, taking them into the index quantification formula and obtain index quantitative value, the index values with the standardized processing are shown in Table 1.

Table 1. Indicator Values of Community A

\begin{tabular}{lllllllr}
\hline & $\delta$ & $J$ & $K$ & $Q$ & $t_{d}$ & $T$ & $\mathrm{D}$ \\
\hline open & 5.90 & 3.13 & 1.10 & 4345 & 0.69 & 151 & 171 \\
closed & 7.12 & 3.79 & 1.09 & 4059 & 0.63 & 138 & 159 \\
\hline
\end{tabular}

\subsection{Weighting Results}

Taking EXCEL and MATLAB as the tool of calculation, according to the standardized indicators index, the combination weighting method is used to get the combined weight of the index which affects the traffic capacity of the community A. The results are shown in Table 2 and Table 3.

Table 2. Index Weights Obtained by Various Weighting Methods of the open community A

\begin{tabular}{ccccccccc}
\hline & & $\delta$ & $J$ & $K$ & $Q$ & $t_{d}$ & $T$ & $\mathrm{D}$ \\
\hline $\mathrm{a}$ & 1 & 0.12 & 0.19 & 0.07 & 0.05 & 0.10 & 0.20 & 0.28 \\
$\mathrm{~b}$ & 2 & 0.14 & 0.13 & 0.13 & 0.14 & 0.18 & 0.13 & 0.16 \\
$\mathrm{c}$ & 3 & 0.14 & 0.13 & 0.13 & 0.14 & 0.17 & 0.13 & 0.16 \\
$\mathrm{~d}$ & 4 & 0.13 & 0.17 & 0.09 & 0.07 & 0.12 & 0.18 & 0.25 \\
\hline
\end{tabular}


Table 3. Index Weights Obtained by Various Weighting Methods of the closed community A

\begin{tabular}{llllllll}
\hline & $\delta$ & $J$ & $K$ & $Q$ & $t_{d}$ & $T$ & $\mathrm{D}$ \\
\hline $\mathrm{a}$ & 0.12 & 0.19 & 0.07 & 0.05 & 0.10 & 0.20 & 0.28 \\
$\mathrm{~b}$ & 0.17 & 0.14 & 0.12 & 0.15 & 0.12 & 0.13 & 0.18 \\
$\mathrm{c}$ & 0.17 & 0.14 & 0.12 & 0.15 & 0.12 & 0.13 & 0.17 \\
$\mathrm{~d}$ & 0.14 & 0.17 & 0.09 & 0.09 & 0.11 & 0.17 & 0.24 \\
\hline
\end{tabular}

Among them, a - relative comparison method, b - variation coefficient method, c - entropy method, d combination weighting method

\subsection{Comprehensive Evaluation Results}

We evaluate and calculate the index value and the weight value of community A, and come to a final result as is shown in Table 4. It can be seen from Table 4 that the comprehensive evaluation index of community A after the opening is larger than that before the opening, which can be considered that the open community is able to enhance traffic capacity in surrounding roads, as well as ease road traffic pressure.

Table 4. Comprehensive evaluation index of community A

\begin{tabular}{lll}
\hline & \multicolumn{2}{l}{ community A } \\
\hline Comprehensive & closed & open \\
evaluation index & 378.32 & 428.55 \\
\hline
\end{tabular}

\section{Conclusion}

The view of this paper is mainly from "Specifications of Urban Road Traffic Planning and Design", which selects the indicators reflecting road space. For example, the road network density, road network connectivity and non-linear coefficient, and there are the main road traffic flow, delay time of intersection, travel time consumption and accessibility that reflect traffic quality. By combining the spearman rank correlation coefficient and correlation degree with the advantages of weighting method, entropy weighting method and variation coefficient method, we make the index weights more reasonable. Connecting standardized index value, we can get the comprehensive score of road traffic capacity surrounded the community before and after the opening, and make a conclusion that the open community can smooth the city capillary, making the city road network extend in all directions, thereby increasing the use of the road, increasing the density of the road network, easing the traffic pressure on the surrounding roads. To some extent, it alleviates the urban traffic congestion, making the road resources be utilized fully, to achieve the aim of resource sharing. Based on the above analysis, it is practical, viable and necessary for us to open the closed area and build an open community, which will become the main development trend of urban construction.

\section{References}

Cheng, D. Y. (2008). Performance Evaluation of Property Insurance Companies Based on Variance Coefficient Approach. Reformation \& Strategy, 2, 128-130. https://doi.org/10.16331/j.cnki.issn1002-736x.2008.02.042

Han, Z. G. (2009). Methods and Applications of Mathematical Modeling. Beijing, China: Higher Education Press.

Li, G., \& Wu, X. Z. (2011). Based on the conclusion of evaluation data standardization research. Mathematics in Practice and Theory, 3, 72-77.

Lu, J., \& Wang, W. (2004). Planning indices system of urban road network. Journal of traffic and Transportation Engineering, 4(4), 62-67. https://doi.org/10.3321/j.issn:1671-1637.2004.04.016

Ma, X. F. (2013). Empirical Study on the Opening Residential Areas of China. Unpublished Master's dissertation, Huazhong University of Science and Technology, Hubei, China.

Shang, Y. H. (2015). Research on the Opening Structural Pattern of the Urban Residential. Unpublished Master's dissertation, Dalian University of Technology, Dalian, China.

Wang, X. H., \& Xing, G. R. (1996).The optimization of comprehensive evaluation of the weighting to determine. Chinese Journal of Health Statistics, 6, 28-30.

Yan, Z. Y., \& Dong, J. Y. (2017). Residential Community is open to the Residential Community and the surrounding traffic capacity of impact analysis. Highways \& Automotive Applications, 1, 37-39. 
Yu, X. Y. (2017). Based on the dimensionless theory to study different types of residential area open to traffic. Science and Technology, 4, 264-265.

Zhan, B., Cai, R. D., Hu, Y. C., \& Cao, M. X. (2016). Residential Community Open-up Strategy Based on Fragility of Urban Road Network. Logistics Technology, 35(7), 98-101. https://doi.org/10.3969/j.issn.1005-152X.2016.07.023

Zhang, L. X., Han, Z. Z., \& Wu, W. Y. (2016). A Comparative Study of Real Estate Appraisal Market Based on AHP and Fuzzy Comprehensive Evaluation Model. Value Engineering, 19, 191-194. https://doi.org/10.14018/j.cnki.cn13-1085/n.2016.19.074

Zhang, P. H., \& Xu, Y. H. (2016). Based on the NS Cellular Automata Traffic Analysis of the Nearby Community Open Road. Journal of Shandong Institute of Commerce and Technology, 6, 105-108. https://doi.org/10.13396/j.cnki.jsict.2016.06.031

Zhang. S., Zhang, M., \& Chi, G. T. (2010). The Science and Technology Evaluation Model Based on Entropy Weight and Empirical Research During the 10th Five-Year of China. Chinese Journal of Management, 1, 34-42. https://doi.org/doi:10.3969/j.issn.1672-884X.2010.01.007

\section{Copyrights}

Copyright for this article is retained by the author(s), with first publication rights granted to the journal.

This is an open-access article distributed under the terms and conditions of the Creative Commons Attribution license (http://creativecommons.org/licenses/by/4.0/). 